\title{
A parameter selection method of the deterministic anti-annealing algorithm for network exploring
}

\author{
Bianfang Chai ${ }^{\mathrm{a}, \mathrm{c}, *}$, Jinghong Wang ${ }^{\mathrm{b}}$, Jian $\mathrm{Yu}^{\mathrm{c}}$ \\ ${ }^{a}$ Department of Information Engineering, Hebei GEO University, Hebei 050031,China \\ ${ }^{b}$ College of Information Technology, Hebei Normal University, Shijiazhuang 050024, China \\ ${ }^{c}$ Beijing Key Lab of Traffic Data Analysis and Mining, Beijing Jiaotong University, Beijing 100044, China
}

\begin{abstract}
The traditional expectation maximization (EM) algorithm for the mixture model can explore the structural regularities of a network efficiently. But it always traps into local maxima. A deterministic annealing EM (DAEM) algorithm is put forward to solve this problem. However, it brings about the problem of convergence speed. A deterministic antiannealing expectation maximization(DAAEM) algorithm not only prevents poor local optima, but also improves the convergence speed. Thus, the DAAEM algorithm is used to estimate parameters of the mixture model. This algorithm always sets its initial parameter $\beta_{0}$ by experience, which maybe get trapped into meaningless results due to too small $\beta_{0}$, or converge to local maxima more frequently due to too large $\beta_{0}$. A parameter selection method for $\beta_{0}$ is designed. In our method, the convergence rate of the DAAEM algorithm for mixture model is first derived from Jacobian matrix of the posterior probabilities. Then the theoretical lower bound of $\beta_{0}$ is computed based on the convergence rate at meaningless points. In our experiments we select $\beta_{0}$ by rounding up the lower bound to the nearest tenth. Experiments on real and synthetic networks demonstrate that the parameter selection method is valid, and the performance of the DAAEM algorithm beginning from the selected parameter is better than the EM and DAEM algorithms for mixture model. In addition, we find that the convergence rate of the DAAEM algorithm is affected by assortative mixing by degree of a network.
\end{abstract}

Keywords: mixture model, community detection, deterministic anti-annealing EM algorithm, convergence rate, Jacobian matrix

\section{Introduction}

Networks have gained significant attention for representing complex systems, and analyzing them helps us understand the systems. When we have no prior on network$\mathrm{s}$, it is necessary to analyze them by automatics. Many analysis techniques for these networks have emerged in the past few years, and community detection [1] is a pop-

${ }^{*}$ Corresponding author at: Department of Information Engineering, Hebei GEO University, Hebei 050031, China.

Email address: chaibianfang@163.com (Bianfang Chai) (Bianfang Chai) ular one. It has become useful for many reasons, such as suppressing the complexity of the whole network and identifying the key nodes in networks, etc.

Up to now, a huge amount of methods for the task of community detection have been developed, including hierarchical clustering, divisive clustering, modularitybased methods, etc. They just focus on detecting tightly connected subgraphs. But complex networks may have many other types of structures, including core-periphery, hierarchical, multipartite structures, or the mixture of them, etc. Recently, some models have been provided to detect a more wide variety of structures besides tightly connected subgraphs. These models are mainly classified 
by two categories. One category is ones based on the stochastic block model (SBM) [2], whose algorithms estimate parameters by the Gibbs sampling method [3], the variational EM algorithm [4, 5, 6], the variational Bayes methods [7], the belief propagation method [8], etc. The time complexities of these algorithms are approximately $O\left(m c^{2}\right)$, where $m$ and $c$ respectively denote the number of edges and clusters. The other category is mixture model [9] for network exploring, whose time complexity is $O(m c)$. By contrast, the EM algorithm for mixture model (EMMM) [9] is more efficient than the algorithms for models based on the SBM.

However, it is well known that the traditional EM algorithm always converges to poor local maxima. The DAEM algorithm [10] has been provided to overcome local maximum problem. It starts with $\beta=\beta_{0} \simeq 0$ and slowly increases $\beta$ to 1 . At each $\beta$, the DAEM algorithm executes the EM algorithm. This increases the convergence time, especially on data with skewed mixing coefficients and large overlap among clusters. The anti-annealing EM algorithm [11] starts from $\beta>1$ and slowly decreases it down to 1 . It improves the speed by restricting the amoun$\mathrm{t}$ of overlaps, but trends to converge to poor local optima more frequently. The DAAEM algorithm [11] not only prevents the EM algorithm from getting trapped into local optima, but also improves the speed of convergence. It starts from a small parameter $\beta=\beta_{0} \simeq 0$, and slowly increases $\beta$ beyond 1 , up to a chosen upper bound, and finally slowly decreases $\beta$ down to 1 .

In order to make the parameter estimating algorithm for mixture model [9] converge to a better local optimum or an approximate global optimum efficiently, we use the framework of the DAAEM algorithm to estimate parameters of mixture model. The DAAEM algorithm for mixture model is noted as DAAEMMM for short. As the DAEM algorithm, the DAAEM algorithm needs to set an initial $\beta_{0}$. In the literature $[10,11]$, the initial $\beta_{0}$ is always set experimentally. Ueda et al[10] confirmed that $\beta_{0}=0.1$ may be small enough, and $\beta$ in the new iteration is set as constant(constant is always set as $1.1 \sim 1.5$ ) times of $\beta$ in the last iteration. Naim et al [11] thought that it was necessary to select a smaller $\beta_{0}$ for complex data, while a larger $\beta_{0}$ for simple data. There is no study on how to set it theoretically. If we set a too small $\beta_{0}$, the DAAEMMM algorithm may divide nodes into meaningless clusters [12], such as the partition that each node belongs to each cluster with equal probabilities. Once the algorithm converges to this case, it is unable to escape from this point in subsequent iterations. If we set a too large $\beta_{0}$, the DAAEMM algorithm is easier to converge to local maxima. From the aspect of convergence property, the algorithm beginning with the selected initial parameter $\beta_{0}$ should not converge to a meaningless clustering result point, which should be an unstable fixed point of the DAAEMMM algorithm.

Some scientists have contributed to study fixed points of algorithms by convergence analysis. The convergence rate is used to measure whether a fixed point is stable. $\mathrm{Pa}-$ rameters embedded in the convergence rate are estimated by making meaningless fixed points be unstable. Hessian matrix and Jacobian matrix are two popular tools to compute the convergence rate. Lei $\mathrm{Xu}$ and Michael I. Jordan presented Hessian matrix of the log-likelihood function for Gaussian mixture with respect to the collection of mixture parameters [13]. Jian Yu et al [14] executed the optimality test by computing Hessian matrix of parameter mapping of the Fuzzy c-means algorithm. The formulas of the Hessian matrix are too complicated to analyze the convergence properties. Jacobian matrix has the same ability as the Hessian matrix to judge whether an algorithm converges to a local maximum. Chaomurilige et al [15] provided a theoretical method for selecting the fuzziness parameter in the Gustafson-Kessel algorithm using Jacobian matrix analysis. It has been demonstrated that Jacobian matrix is a more effective tool for convergence analysis, which is used to select the parameters of an algorith$\mathrm{m}$ by making the convergence rate at meaningless fixed points be not less than 1. But there is no study on how to select $\beta_{0}$ parameter of the DAAEM or DAEM algorithm using Jacobian matrix. Here we provide a theoretical parameter selection method for the initial parameter $\beta_{0}$ of the DAAEMMM algorithm, which is also suitable to the DAEM algorithm for mixture model (DAEMMM). In our method, the convergence rate of the DAAEMM$\mathrm{M}$ algorithm is computed based on the Jacobian matrix of the posterior probabilities. Then, a theoretical lower bound of parameter $\beta_{0}$ is captained by analyzing the stability of fixed points according to the convergence rate. The initial parameter $\beta_{0}$ is set according to this theoretical lower bound value. In our experiments, $\beta_{0}$ are selected by rounding up the theoretical lower bound to the nearest tenth.

In this paper, we first use the DAAEM algorithm to esti- 
mate parameters of mixture model for network exploring. Then we design a method to get the theoretical minimal $\beta_{0}$ for the DAAEMMM algorithm based on Jacobin matrix analysis. In outline, the paper is organized as follows. In section 2, we give a brief review of the EMMM algorithm for exploratory analysis in networks. In section 3, we analyze the disadvantages of the EMMM algorithm on several real networks, and then present a DAAEMM$\mathrm{M}$ algorithm for general structure detection. In section 4, we design a theoretical parameter selection method for $\beta_{0}$ based on the convergence rate. Finally, experiments on synthetic and real networks validate the selected parameter according to the theoretical lower bound of $\beta_{0}$ from our provided method and demonstrate the performance of the DAAEMMM algorithm. In addition, we test the relations between the convergence rate and the structure of a network measured by assortative mixing by degree. Finally, the conclusions are stated.

\section{The EM algorithm of mixture model for network exploring}

In this section, we first describe the mixture model for exploratory analysis in networks. Then the EM algorithm of its parameter estimation (EMMM) in directed networks [9] is inferred.

Structure detection based on mixture model [9] aims to deduce the assignments of nodes in a network by fitting a model to an observed network. Here we focus on the mixture model for directed networks, and it is easy to extend to the case of undirected or weighted networks. A network with $N$ nodes is represented by an adjacency matrix $A$ with element $A_{i j}=1$ if there is an edge from node $i$ to node $j$ and 0 otherwise. Suppose that nodes of a network fall into $c$ communities and model parameters are specified as the triplet $\left(\left\{g_{i}\right\},\left\{\pi_{r}\right\},\left\{\theta_{r i}\right\}\right)$, where the hidden variable $g_{i}$ indicates the group assignment of node $i, \pi_{r}$ the fraction of nodes in group $r$, and $\theta_{r i}$ the probability that there is a directed edge from nodes of group $r$ to node $i$. The model parameters $\left\{\pi_{r}\right\}$ and $\left\{\theta_{r i}\right\}$ satisfy the normalization conditions $\sum_{r=1}^{c} \pi_{r}=1$ and $\sum_{i=1}^{N} \theta_{r i}=1$.

Assume that edges of a network are generated by a mixture of underlying probability distribution. Each edge from $i$ to $j$ is generated independently. First, the begin node $i$ of a edge $\left\langle i, j>\right.$ selects its group $g_{i}$ by a probability $\pi_{g_{i}}$. Then a node $i$ in group $g_{i}$ links to end node $j$ by a probability $\theta_{g_{i} j}$. The likelihood of an observed network $A$ can be written as

$$
\begin{aligned}
\operatorname{Pr}(A \mid \pi, \theta) & =\prod_{i j} \operatorname{Pr}\left(A_{i j} \mid \pi, \theta\right) \\
& =\prod_{i} \sum_{r=1}^{c}\left\{\operatorname{Pr}\left(g_{i}=r \mid \pi\right) \prod_{j} \operatorname{Pr}\left(A_{i j} \mid g_{i}=r, \theta\right)\right\} \\
& =\prod_{i} \sum_{r=1}^{c}\left[\pi_{r} \prod_{j}\left(\theta_{r j}\right)^{A_{i j}}\right] .
\end{aligned}
$$

The logarithm of the likelihood in Eq. (1) is

$$
\begin{aligned}
L & =\log \operatorname{Pr}(A \mid \pi, \theta) \\
& =\sum_{i} \log \sum_{r=1}^{c}\left[\pi_{r} \prod_{j}\left(\theta_{r j}\right)^{A_{i j}}\right] .
\end{aligned}
$$

We often use the EM algorithm to estimate the parameters by maximizing the log-likelihood $L$. The lower bound of $L$ can be computed by Jesen inequality from Eq. (2), noted as $\bar{L}$.

$$
\bar{L}=\sum_{i r} q_{i r}\left[\log \pi_{r}+\sum_{j} A_{i j} \log \theta_{r j}\right]-\sum_{i r} q_{i r} \log q_{i r},
$$

where $q_{i r}$ is the posterior probability that node $i$ belongs to cluster $r$, and it satisfies the normalization condition $\sum_{r} q_{i r}=1$.

By introducing the Lagrange multiplier to incorporate the constraint $q$ in E step, the objective function becomes

$$
\overline{L^{\prime}}=\bar{L}+\lambda\left(1-\sum_{r} q_{i r}\right)
$$

By letting the derivative of $\overline{L^{\prime}}$ by $q_{i r}$ be zero in E step, the updating equation for $q_{i r}$ is inferred as follows:

$$
q_{i r}=\frac{\operatorname{Pr}\left(A_{i,}, g_{i}=r \mid \pi, \theta\right)}{\operatorname{Pr}\left(A_{i .} \mid \pi, \theta\right)}=\frac{\pi_{r} \prod_{j} \theta_{r j}^{A_{i j}}}{\sum_{s} \pi_{s} \prod_{j} \theta_{s j}^{A_{i j}}} .
$$

By introducing the Lagrange multipliers to incorporate the normalization conditions $\sum_{r} \pi_{r}=1$ and $\sum_{j} \theta_{r j}=1$, the objective function becomes

$$
L L=\bar{L}+\alpha\left(1-\sum_{r} \pi_{r}\right)+\sum_{j} \beta_{j}\left(1-\sum_{j} \theta_{r j}\right)
$$


By letting the derivatives of $L L$ by $\pi_{r}$ or $\theta_{r j}$ be 0 in $M$ step, the updating equations of model parameters are gotten as follows:

$$
\pi_{r}=\frac{1}{N} \sum_{i} q_{i r}, \quad \theta_{r j}=\frac{\sum_{i} A_{i j} q_{i r}}{\sum_{i} d_{i} q_{i r}},
$$

where $d_{i}$ denotes the out-degree of node $i$.

In terms of the framework of the EM algorithm, the EM algorithm of mixture model updates $q$ and $\pi, \theta$ by Eq. (5) and Eq. (7) iteratively until the algorithm converges.

\section{Deterministic anti-annealing expectation maxi- mization algorithm for mixture model}

In this section, we first illustrate that the EM algorithm for mixture model always converges to many poor local maxima. Then the deterministic anti-annealing expectation maximization algorithm is used to estimate parameters of mixture model.

In order to analyze the convergence property of the EM algorithm for mixture model in the task of general structure detection, we test the converged log-likelihood values after running the EM algorithm for 50 times on several small networks, including the karate network, the dolphin network, and the adjacency network. The EM algorithm is restarted for 50 times. Each EM algorithm iterates until the relative difference of log-likelihoods between two successive iterations is less than a threshold $10^{-10}$. Test$\mathrm{s}$ show that the EM algorithm for mixture model always converges to several local optima. The results of tests are listed in Tables 1, 2, and 3. From the results, we find the EM algorithm may converge to more local optima, especially when a network becomes more complex. This demonstrates that the EM algorithm for mixture model always converges to many poor local maxima [16].

Deterministic anti-annealing expectation maximization (DAAEM) algorithm is a well-known algorithm for maximum likelihood estimation. It not only overcomes local optimum problem of the traditional EM algorithm, but also improves the speed of convergence. It is used to estimate parameters of mixture model. As the framework of the DAEM algorithm [10], the DAAEMMM algorith$\mathrm{m}$ introduces a parameter $\beta$ corresponding to the inverse temperature, and the traditional EM algorithm is a special case of the DAAEMMM algorithm at the temperature $\beta=1$. The DAAEMMM algorithm adds a temperature parameter $\beta$ in the posterior probability of $q$ in Eq.(5),

\begin{tabular}{ccc}
\hline log-likelihood & average-iterations & times \\
\hline-469.883163 & 51 & 30 \\
-470.871518 & 55 & 8 \\
-481.885278 & 30 & 12 \\
\hline
\end{tabular}

Table 1: Results on the karate network with 34 nodes and 78 edges. The label 'log-likelihood' denotes the log likelihood value that the EM algorithm converges. The label 'average-iterations' denotes the average number that all the restarts iterate for each converged value. The label 'times' denotes the number of all the 50 restarts for each log-likelihood value.

\begin{tabular}{ccc}
\hline log-likelihood & average-iterations & times \\
\hline-1127.356946 & 30 & 43 \\
-1134.89884 & 87 & 2 \\
-1195.712438 & 66 & 3 \\
-1205.407540 & 35 & 2 \\
\hline
\end{tabular}

Table 2: Results on the dolphin network with 62 nodes and 159 edges. The labels of the table are the same as Table 1.

\begin{tabular}{ccc}
\hline log-likelihood & average-iterations & times \\
\hline-3615.218331 & 80 & 21 \\
-3618.810749 & 71 & 4 \\
-3621.311188 & 71 & 9 \\
-3622.883832 & 223 & 4 \\
-3624.923492 & 138 & 3 \\
-3631.840472 & 157 & 5 \\
-3652.077227 & 83 & 4 \\
\hline
\end{tabular}

Table 3: Results on the adjnoun network with 112 nodes and 425 edges. The labels of the table are the same as Table 1.

\begin{tabular}{cccc}
\hline network & log-likelihood & average-iterations & times \\
\hline dolphin & -1127.356946 & 117 & 50 \\
karate & -469.883163 & 69 & 50 \\
adjnoun & -3611.55376 & 418 & 50 \\
\hline
\end{tabular}

Table 4: The DAAEMMM algorithm is restarted for 50 times in the three networks. The labels of the table are the same as Table 1. 
the new posterior probabilities parameterized by $\beta$ can be rewritten as:

$$
p_{i r}=\frac{\left(\pi_{r} \prod_{j} \theta_{r j}^{A_{i j}}\right)^{\beta}}{\sum_{s}\left(\pi_{s} \prod_{j} \theta_{s j}^{A_{i j}}\right)^{\beta}},
$$

where $1 / \beta$ is the corresponding temperature.

When the posterior probabilities are known, the model parameters are estimated using this posterior probabilities in the same manner as the EM algorithm. The algorithm starts from $\beta=\beta_{0}<<1$ (high temperature) and slowly increases $\beta$ up to $\beta_{\max }>1$ and then decreases back to $\beta=1$. At the final temperature the DAAEMMM algorithm is similar to the traditional EM algorithm. The DAAEMM$\mathrm{M}$ algorithm iterates until convergence just like the traditional EM algorithm. The procedure of the DAAEMMM algorithm is rewritten as Algorithm1.

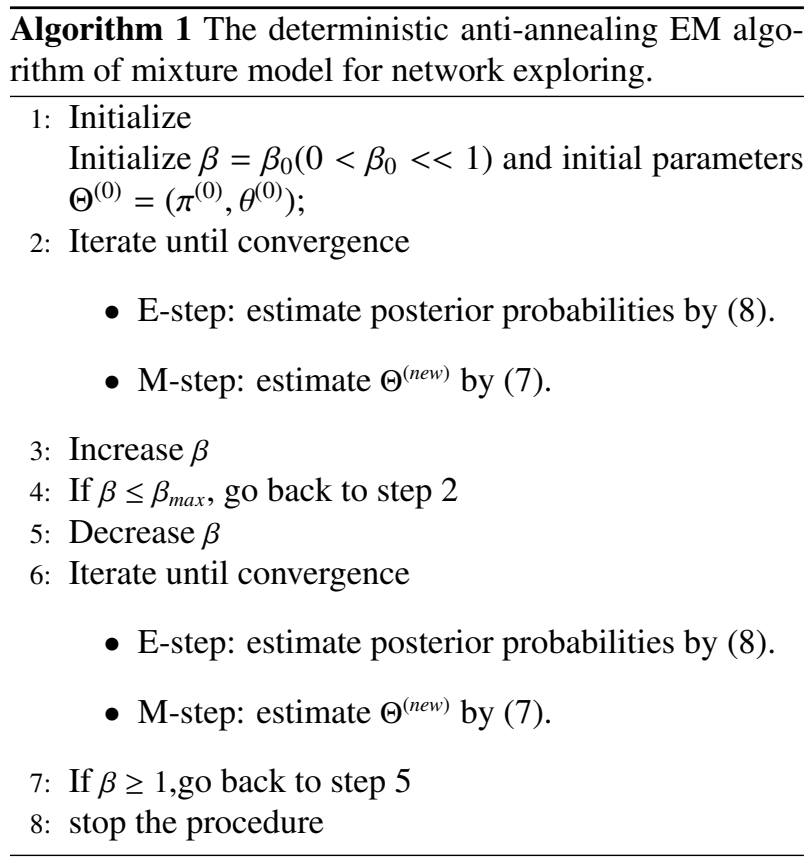

In the DAAEMMM algorithm, the problem of maximizing the log-likelihood function is reformulated as the problem of minimizing a free energy function. The algorithm begins at high temperature with high entropy, and high temperature corresponds to a small initial $\beta=\beta_{0}$. If $\beta_{0} \longrightarrow 0$, the posterior membership probabilities of each node become uniform on all clusters. The partition matrix of this case is noted as $\left[\frac{1}{c}\right]_{N \times c}$, which fails to partition nodes of a network. Once the algorithm converges to this point, the algorithm will always output this meaningless result in the subsequent iterations and $\left[\frac{1}{c}\right]_{N \times c}$ will be a fixed point of the algorithm. If this point is not stable, the algorithm is able to escape from it. Otherwise, the algorithm will be trapped into this meaningless result. Thus, the initial $\beta_{0}$ should be set properly, such that the algorith$\mathrm{m}$ can avoid meaningless clustering results. A proper $\beta_{0}$ can make the DAAEMMM algorithm not stable at meaningless results. But $\beta_{0}$ is always given by experience, and no research tells us how to set this free parameter $\beta_{0}$ theoretically. In the next section, we will provide a parameter selection method, which selects a $\beta_{0}$ theoretically.

\section{A parameter selection method for the DAAEMMM algorithm based on the convergence rate}

The principle of our parameter selection is to make the clustering algorithm unstable at meaningless clustering fixed points. The stability of an algorithm can be measured by its convergence rate. Our method firstly derives the convergence rate of the DAAEMMM algorithm based on Jacobain Matrix. Then the convergence rate at a meaningless clustering fixed point is computed. By making this convergence rate be not less than 1 we can get a theoretical lower bound of $\beta_{0}$. Theoretically, any $\beta_{0}$ that is not less than the theoretical lower bound is ok so that the DAAEMMM algorithm avoids meaningless clustering results.

Partition matrix of the DAAEMMM algorithm is the posterior membership probabilities $\left\{p_{i r}\right\}(i \in(1, \ldots, N), r \in$ $(1, \ldots, c))$. It is easy to judge whether a clustering result is meaningful according to partition matrix. In the following, we first define two formalized meaningless clustering results according to partition matrix.

Meaningless partition: $U_{\pi}=\left[\pi_{1}, \pi_{2}, \cdots, \pi_{c}\right]^{T} \otimes \mathbf{1}_{1 \times N}$ is called meaningless partition, where $\otimes$ represents Kronecker product, 1 denotes the vector of all 1's. This partition represents that all the nodes belong to the cluster $r(r=1, \ldots, c)$ with the same probability $\pi_{r}$.

Absolute meaningless partition: $U_{\frac{1}{c}}=\left[\frac{1}{c}\right]_{c \times N}$ is called absolute meaningless partition. This partition represents that all the nodes belong to each cluster with the 
same probability $\frac{1}{c}$. Absolute meaningless partition is a special case of meaningless partition.

Once the DAAEMMM algorithm outputs partitions $U_{\pi}$ or $U_{1}$, it will converge to the same partition in the successive iterations. Thus, the two partitions should be avoided. It can be controlled by letting the DAAEMMM algorith$\mathrm{m}$ unstable at these points. Researchers usually use the method of convergence analysis to judge whether an algorithm is stable at some point. And existing methods of convergence analysis need to compute the convergence rate of an algorithm.

Here we just provide how to compute the convergence rate of the DAAEMMM algorithm in the case of directed networks, which is easy to be extended to undirected networks. In the literature two kinds of tools are usually used to compute the convergence rate, including Hessian matrix and Jacobian matrix. By Hessian matrix [17, 18], it is necessary to compute Hessian matrix of all model parameters in Eq. (7) of the DAEMMM algorithm. The different formulas of Hessian matrix of the parameters ad$\mathrm{d}$ the difficulty to analyze the convergence rate. Jacobian matrix has the same ability to analyze the convergence property of an algorithm. In addition, Jacobian matrix is easier to compute than Hessian matrix. Chaomurilige, $\mathrm{Yu}$ and Yang have used Jacobian matrix to compute the convergence rate of the GK algorithm [15].

According to Olver's corollary [19], if an algorithm converges to a stable fixed point $p^{*}$, the spectral radius (or the largest eigenvalue) of the Jacobian matrix at the point $p^{*}$ should be less than 1 .

Here, we compute the convergence rate of the DAAEMMM algorithm based on Jacobian matrix. Jacobian matrix is computed by $f^{\prime}\left(p^{*}\right)=\left.\frac{\partial f(p)}{\partial p}\right|_{p=p^{*}}$, where $\left\{p^{0}, p^{1}, p^{2}, \ldots, p^{n}, \ldots, p^{*}\right\}$ is a parameter convergence sequence. In order to get the Jacobian matrix, we first define the parameter map of the DAAEMMM algorithm.

The iterative formula of the DAAEMMM algorithm can be formatted as a map $p^{(t+1)}=F^{(\beta)}\left(p^{(t)}\right)$ in the following.

$$
p_{i r}^{(t+1)}=F^{(\beta)}\left(p_{i r}^{(t)}\right)=\frac{\left(\pi_{r}^{(t+1)} \prod_{j}\left(\theta_{r j}^{(t+1)}\right)^{A_{i j}}\right)^{\beta}}{\sum_{s}\left(\pi_{s}^{(t+1)} \prod_{j}\left(\theta_{s j}^{(t+1)}\right)^{A_{i j}}\right)^{\beta}},
$$

where

$$
\pi_{r}^{(t+1)}=\frac{1}{N} \sum_{i} p_{i r}^{(t)}, \quad \theta_{r j}^{(t+1)}=\frac{\sum_{i} A_{i j} p_{i r}^{(t)}}{\sum_{i} d_{i} p_{i r}^{(t)}}
$$

$\left\{p^{(t)}, t=0,1, \ldots, n, \ldots\right\}$ is called a convergence sequence of the DAAEMMM algorithm. The sequence starts from the point $p^{0}$, and will converge to a point $p^{*}$. The convergence point is a fixed point, i.e., $p^{*}=F^{\beta}\left(p^{*}\right)$. It is easy to prove $\left[\pi_{1}, \pi_{2}, \cdots, \pi_{c}\right]^{T} \otimes \mathbf{1}_{1 \times N}$ and $\left[c^{-1}\right]_{c \times N}$ are fixed points of the DAAEMMM algorithm. $\left[c^{-1}\right]_{c \times N}$ is a special case of $\left[\pi_{1}, \pi_{2}, \cdots, \pi_{c}\right]^{T} \otimes \mathbf{1}_{1 \times N}$, and we just consider the computing on $p^{*}=U_{\pi}=\left[\pi_{1}, \pi_{2}, \cdots, \pi_{c}\right]^{T} \otimes \mathbf{1}_{1 \times N}$. The algorithm should not converge to these meaningless points stably, which is realized by letting the largest eigenvalue of the Jacobian matrix $\left.\left(\partial F^{(\beta)}\left(p_{i r}\right) / \partial p_{j s}\right)\right|_{p=p^{*}}$ at the point be not less than 1. The DAAEMMM algorithm has the constraint $\sum_{i=1}^{c} p_{i r}=1$, then $p_{i c}=1-\sum_{i=1}^{c-1} p_{i r}$. The dimension of the Jacobian matrix $\left[\partial F^{(\beta)}\left(p_{i r}\right) / \partial p_{j s}\right]_{i j r s}$ is $(c-1) \times(c-1) \times N \times N(i, j \in\{1, \ldots, N\}, r, s \in\{1, \ldots, c-1\})$. The element at the $(r-1) \times N+i$ row and the $(s-1) \times N+j$ column of the Jacobian matrix can be represented by a unifying expression in Lemma 1 as follows:

Lemma 1 For $i=1, \ldots, N, r=1, \ldots, c-1$ and $j=$ $1, \ldots, N, s=1, \ldots, c-1$, each element $\frac{\partial F^{(\beta)}\left(p_{i r}\right)}{\partial p_{j s}}$ of Jacobian matrix $\frac{\partial F^{(\beta)}(p)}{\partial p}$ is computed by the following equation.

$$
\begin{aligned}
& \frac{\partial F_{i r}^{(\beta)}}{\partial p_{j s}} \\
& =\beta \times \delta_{r s}\left(\frac{p_{i r}}{N \pi_{r}}+p_{i r} \sum_{l=1}^{N} \frac{A_{i l}}{\theta_{r l}} \frac{A_{j l}-d_{j} \theta_{r l}}{\sum_{k=1}^{N} d_{k} p_{k r}}\right) \\
& -p_{i r} \times \beta \times\left(\frac{p_{i s}}{N \pi_{s}}+p_{i s} \sum_{l=1}^{N} \frac{A_{i l}}{\theta_{s l}} \frac{A_{j l}-d_{j} \theta_{s l}}{\sum_{k=1}^{N} d_{k} p_{k s}}\right) \\
& -p_{i r} \times \beta \times\left(-\frac{p_{i c}}{N \pi_{c}}-p_{i c} \sum_{l=1}^{N} \frac{A_{i l}}{\theta_{c l}} \frac{A_{j l}-d_{j} \theta_{c l}}{\sum_{k=1}^{N} d_{k} p_{k c}}\right),
\end{aligned}
$$

where $\delta_{r s}=\left\{\begin{array}{ll}1 & \mathrm{r}=\mathrm{s} \\ 0 & \mathrm{r} !=\mathrm{s}\end{array}\right.$ is a kronecker delta function, and $\frac{\partial \theta_{r l}}{\partial p_{j s}}=\frac{A_{j l}-d_{j} \theta_{r l}}{\sum_{k=1}^{N} d_{k} p_{k r}}$, and $d_{k}=\sum_{l=1}^{N} A_{k l}$.

Proof. The DAAEMMM algorithm is formatted as Eq.(9). The Jacobian matrix of the algorithm is computed 
by taking the derivatives of $F_{i r}^{\beta}$ with respective to $p_{j s}$ for $j=1, \ldots, N$ and $s=1, \ldots, c-1$ as follows:

$$
\begin{aligned}
& \frac{\partial F_{i r}^{(\beta)}}{\partial p_{j s}}=\frac{\partial\left(\frac{\left(\pi_{r} \prod_{l} \theta_{r l}^{\left.A_{i l}\right)^{\beta}}\right.}{\sum_{s}\left(\pi_{s} \prod_{l} \theta_{s l}^{\left.A_{i l}\right)^{\beta}}\right.}\right)}{\partial p_{j s}} \\
& =\delta_{r s} \frac{\beta\left(\pi_{r} \prod_{l} \theta_{r l}^{A_{i l}}\right)^{(\beta-1)}}{\sum_{s}\left(\pi_{s} \prod_{l} \theta_{s l}^{A_{i l}}\right)^{\beta}}\left(\frac{\partial\left(\pi_{r} \prod_{l} \theta_{r l}^{A_{i l}}\right)}{\partial\left(p_{j s}\right)}\right) \\
& -\frac{\frac{\partial\left(\sum_{s}\left(\pi_{s} \prod_{l} \theta_{s l}^{A_{i l} \beta^{\beta}}\right)\right.}{\partial\left(p_{j s}\right)}}{\left(\sum _ { s } \left(\pi_{s} \prod_{l} \theta_{s l}^{\left.\left.A_{i l}\right)^{\beta}\right)^{2}}\right.\right.} \\
& =\delta_{r s} \frac{\beta\left(\pi_{r} \prod_{l} \theta_{r l}^{A_{i l}}\right)^{(\beta-1)}}{\sum_{s}\left(\pi_{s} \prod_{l} \theta_{s l}^{A_{i l}}\right)^{\beta}}\left(\frac{\partial \pi_{r}}{\partial p_{j s}} \frac{\pi_{r} \prod_{l} \theta_{r l}^{A_{i l}}}{\pi_{r}}\right. \\
& \left.+\pi_{r} \prod_{l} \theta_{r l}^{A_{i j}} \sum_{l=1}^{N} \frac{A_{i l}}{\theta_{r l}} \frac{\partial \theta_{r l}}{\partial p_{j s}}\right)-\frac{\frac{\partial\left(\left(\pi_{j} \prod_{l} \theta_{j l}^{A_{i l}}\right)^{\beta}+\left(\pi_{c} \prod_{l} \theta_{c l}^{A_{i l}}\right)^{\beta}\right)}{\partial\left(p_{j s}\right)}}{\left(\sum_{s}\left(\pi_{s} \prod_{l} \theta_{s l}^{A_{i l}}\right)^{\beta}\right)^{2}} \\
& =\beta \times \delta_{r s}\left(\frac{p_{i r}}{N \pi_{r}}+p_{i r} \sum_{l=1}^{N} \frac{A_{i l}}{\theta_{r l}} \frac{A_{j l}-d_{j} \theta_{r l}}{\sum_{k=1}^{N} d_{k} p_{k r}}\right) \\
& -\frac{\frac{\partial\left(\left(\pi_{j} \prod_{l} \theta_{j l}^{\left.\left.A_{i l}\right)^{\beta}\right)}\right.\right.}{\partial\left(p_{j s}\right)}}{\left(\sum _ { s } \left(\pi_{s} \prod_{l} \theta_{s l}^{\left.\left.A_{i l}\right)^{\beta}\right)^{2}}\right.\right.}-\frac{\frac{\partial\left(\left(\pi_{c} \prod_{l} \theta_{c l}^{\left.A_{i l} \beta^{\beta}\right)}\right.\right.}{\partial\left(p_{j s}\right)}}{\left(\sum_{s}\left(\pi_{s} \prod_{l} \theta_{s l}^{A_{i l}}\right)^{\beta}\right)^{2}} \\
& =\beta \times \delta_{r s}\left(\frac{p_{i r}}{N \pi_{r}}+p_{i r} \sum_{l=1}^{N} \frac{A_{i l}}{\theta_{r l}} \frac{A_{j l}-d_{j} \theta_{r l}}{\sum_{k=1}^{N} d_{k} p_{k r}}\right) \\
& -p_{i r} \times \beta \times\left(\frac{p_{i s}}{N \pi_{s}}+p_{i s} \sum_{l=1}^{N} \frac{A_{i l}}{\theta_{s l}} \frac{A_{j l}-d_{j} \theta_{s l}}{\sum_{k=1}^{N} d_{k} p_{k s}}\right) \\
& -p_{i r} \times \beta \times\left(-\frac{p_{i c}}{N \pi_{c}}-p_{i c} \sum_{l=1}^{N} \frac{A_{i l}}{\theta_{c l}} \frac{A_{j l}-d_{j} \theta_{c l}}{\sum_{k=1}^{N} d_{k} p_{k c}}\right) \text {. }
\end{aligned}
$$

From the analysis above, we know the DAAEMMM algorithm should not be stable at the fixed point $p^{*}=U_{\pi}$. According to Olvers corollary, the largest eigenvalue of $\left.\frac{\partial F^{(\beta)}(p)}{\partial p}\right|_{p^{*}=U_{\pi}}$ should not be less than 1. Each element of $\left.\frac{\partial F^{(\beta)}(p)}{\partial p}\right|_{p^{*}=U_{\pi}}$ is computed as follows:

$$
\left.\frac{\partial F\left(p_{i r}\right)}{\partial p_{j s}}\right|_{p^{*}=U_{\pi}}=\beta \times \delta_{r s}\left(\frac{1}{N}-\frac{d_{i} d_{j}}{\sum_{k=1}^{N} d_{k}}+\sum_{l=1}^{N} \frac{A_{i l} A_{j l}}{\sum_{k=1}^{N} A_{k l}}\right) .
$$

If the largest eigenvalue of the Jacobian matrix by Eq. (13) is not less than 1, i.e., $\lambda_{\max }\left(\left.\frac{\partial F(p)}{\partial p}\right|_{p^{*}=U_{\pi}}\right)>=1$, the algorithm will be unstable at the point $p^{*}=U_{\pi}$ and can not converge to the corresponding point. In experiments, we can demonstrate the largest eigenvalue of Jacobian matrix by Eq. (13) at $\beta=1$ are larger than 1 for several networks. That means the original EM algorithm is unable to converge to $U_{\pi}$ or $\left[\frac{1}{c}\right]_{(N * c)}$. As $U_{\pi}$ or $\left[\frac{1}{c}\right]_{(N * c)}$ are unstable fixed points, we can set starting values that are perturbed randomly a small distance from them. This explains theoretically the reason that initial parameters are set as equal probabilities $q_{i r}=\frac{1}{c}$ for $r=1, \ldots, c$ and $i=1, \ldots, N$ (namely $\pi_{r}=\frac{1}{c}$ and $\theta_{r i}=\frac{1}{N}$ ) with a small perturb in the original EM algorithm of mixture model for network exploring [9].

For a smaller $\beta_{0}$, the largest eigenvalue of the Jacobian matrix by Eq. (13) may be less than 1. So we must select a proper $\beta_{0}$ and guarantee the largest eigenvalue of the Jacobian matrix by Eq. (13) is not less than 1. The matrix $\left[\left(\delta_{r s}\left(\frac{1}{N}-\frac{d_{i} d_{j}}{\sum_{k=1}^{N} d_{k}}+\sum_{l=1}^{N} \frac{A_{i l} A_{j l}}{\sum_{k=1}^{N} A_{k l}}\right)\right)\right]_{(c-1) \times(c-1) \times N \times N}($ noted as $A)$ can be seen as a $(c-1) \times(c-1)$ dimension super-matrix $B$. And each element of $B$ is a matrix with $N \times N$ dimension. Only the diagonal elements $B_{r r}(r=1, \ldots, c-1)$ of the matrix $B$ are nonzero. In addition, all the non-diagonal matrices are equal. Thus, the largest eigenvalue of the Jacobian matrix by Eq.((13)) can be computed according to the largest eigenvalue of a diagonal element $B_{11}$, which is a $N \times N$ matrix . A theoretical smallest value of $\beta_{0}$ for a network must not be less than $\beta_{\text {theoretical }}=1 / \lambda_{\max }\left(B_{11}\right)$. When we run the DAAEMMM algorithm, the initial $\beta_{0}$ for a network should not be less than this theoretical value $\beta_{\text {theoretical }}$. Otherwise, the algorithm is easy to output meaningless partitions. In our experiments, we round up the lower bound $\beta_{\text {theoretical }}$ to nearest tenth, and use this value as $\beta_{0}$ of the DAAEMMM algorithm.

When we have computed the largest eigenvalue of Jacobian matrix, how to get the convergence rate of the DAAEMMM algorithm?

The DAAEMMM algorithm can be formatted as a mapping $p \rightarrow F(p)$ such that $F\left(p^{t-1}\right)=p^{t}$ with an iteration sequence $\left\{p^{t}, t \geq 0\right\}$. Suppose the mapping is differentiable in a neighborhood of $p^{*}$, a Taylor expansion is written as

$$
p^{t+1}-\left.p^{*} \approx\left(p^{t}-p^{*}\right) \frac{\partial F^{(\beta)}(p)}{\partial p}\right|_{p=p^{*}}
$$

Thus, the DAAEMMM algorithm is a linear iteration with the rate $\left.\frac{\partial F^{(\beta)}(p)}{\partial p}\right|_{p=p^{*}}$. The convergence rate should be related to the largest eigenvalue of Jacobian matrix $\frac{\partial F^{(\beta)}(p)}{\partial p}$, 


\begin{tabular}{cccc}
\hline networks & nodes & edges & groups \\
\hline football & 115 & 613 & 12 \\
lesmis & 77 & 254 & 11 \\
risk & 42 & 83 & 6 \\
karate & 34 & 78 & 2 \\
dolphin & 62 & 159 & 2 \\
adjnoun & 112 & 425 & 2 \\
polbook & 105 & 441 & 3 \\
\hline
\end{tabular}

Table 5: The properties of several small real networks with ground truth The label of 'networks' represents the name of a network. The labels of 'nodes', 'edges', and 'groups' represent the corresponding numbers.

\begin{tabular}{cccc}
\hline networks & nodes & edges & groups \\
\hline Wiki-Vote & 7115 & 103,689 & 20 \\
socSlash & 82,168 & 948,464 & 5 \\
Web & 281,903 & $2,312,497$ & 5 \\
\hline
\end{tabular}

Table 6: The properties of several large real networks without ground truth. The labels are the same as Table 5.

which can be seen as the convergence rate.

\section{Experiments}

In this section, we first validate the theoretical lower bound $\beta_{\text {theoretical }}$ of initial parameter computed by the proposed parameter selection method for the DAAEMMM algorithm by experiments. And then we demonstrate the performance of the DAAEMMM algorithm which starts from $\beta_{0}$ selected by rounding up $\beta_{\text {theoretical }}$ to the nearest tenth. In addition, we study relations between convergence rate and the structure of networks. Before we show the performance of the related methods, we first introduce network data and measures in the experiments.

\subsection{Synthetic networks and real networks}

Our tests are done on real networks and synthetic networks.

Several real networks ${ }^{1}$ with ground truth are shown in Table 5. Several real networks without ground truth are shown in Table 6.

We generate two groups of synthetic networks. One group of synthetic networks are generated by the SBM.

\footnotetext{
${ }^{1}$ http://www-personal.umich.edu// mejn/netdata/.
}

Similar to mixture model, it can generate networks with a wide variety of structural types. Since the SBM is easy to set model parameters to generate a network, our synthetic networks are generated by the SBM characterized by a matrix of a link probability. For simplicity, we just consider two kinds of values of the matrix: the probability within clusters and the probability between clusters. Edges between two nodes in the same cluster are linked with a probability $\lambda$ and edges between nodes in different clusters are linked with a probability $\epsilon$. This simplified SBM is named as affiliation model in Mixer R package ${ }^{2}$. One group of synthetic networks with assortative and disassortative structure are generated by this package. These networks are divided by 10 clusters with $\lambda=0.7, \epsilon=0.3$. The number of nodes changes from 200 to 6400 , and the number of edges changes from 3032 to $3,073,451$, shown in Table 7. The other group of synthetic networks are generated by the LFR benchmark ([20]), shown in Table 11. The number of the vertices $N$ is set as 5000. The exponents of the power law distributions of degree and community sizes are set by $\gamma=2$ and $\beta=1$, respectively. The average of the degree is 38 . Each vertex shares a fraction $1-\mu$ of its edges with the other vertices of its community and a fraction $\mu$ with the vertices of the other communities. $\mu$ is the mixing parameter.

\subsection{Measures and experiment sets}

In order to test the performance of the DAAEMMM algorithm, we measure it by accuracy (noted as acc), Normalized Mutual Information (noted as nmi) at several networks with ground truth.

A community structure is $C=\left(C_{1}, C_{2}, \ldots, C_{K}\right)$, where $C_{k}$ contains a set of nodes that are in the $k$ th community. $C^{\prime}=\left(C_{1}^{\prime}, C_{2}^{\prime}, \ldots, C_{K}^{\prime}\right)$ represents a community structure given by an algorithm. The NMI $P_{n m i}$ is defined by:

$$
P_{n m i}\left(C, C^{\prime}\right)=\frac{2 M_{I}\left(C, C^{\prime}\right)}{H(C)+H\left(C^{\prime}\right)}
$$

where $H(C)$ and $H\left(C^{\prime}\right)$ are the entropies of partitions $C$ and $C^{\prime}$, respectively, and $M_{I}\left(C, C^{\prime}\right)$ is the mutual information between the two partitions.

Given each node $i$, its true label $s_{i}$ and the assigned label $r_{i}$ obtained from an algorithm, the ACC of the partition

${ }^{2}$ http://cran.r-project.org/web/packages/mixer/ 
$P_{a c c}$ is defined as follows:

$$
P_{a c c}=\frac{\sum_{i \in N_{s e t}} \delta\left(s_{i}, p_{\text {map }}\left(r_{i}\right)\right)}{\left|N_{\text {set }}\right|}
$$

where $\left|N_{\text {set }}\right|$ is the number of the nodes set $N_{\text {set }}, \delta(x, y)$ is a delta function that is 1 if $x=y$ and is 0 otherwise, and $p_{\text {map }}\left(r_{i}\right)$ is a permutation mapping function that maps the label $r_{i}$ of node $i$ to the corresponding label in the ground truth.

For the two measures, i.e., nmi and acc, the larger the value is, the better the performance is.

In order to test the relations between the convergence rate and the structure of a network, it is necessary to measure the structure of a network. Since there are terms of degree of nodes in Jacobian matrix, we use assortative mixing by degree to measure the structure of a network. Assortative mixing by degree measures the tendency of vertices to connect others with degrees that are similar to their own. It is defined by assorDegee = $\frac{\sum_{i, j}\left(A_{i j}-k_{i} k_{j} / 2 m\right) k_{i} k_{j}}{\sum_{i, j}\left(k_{i} \delta_{i j}-k_{i} k_{j} / 2 m\right) k_{i} k_{j}}$ with values from -1 to $1 . \delta_{i j}=1$ if node $i$ is in the same cluster as node $j$, otherwise $\delta_{i j}=0$. $k_{i}$ is the degree of node $i$.

All the algorithms terminate when they satisfy the stopping criterion: $\left|L\left(\theta^{t+1}\right)-L\left(\theta^{t}\right)\right| / L\left(\theta^{t+1}\right)<\tau$, where $L\left(\theta^{t}\right)$ represents the log-likelihood value computed by Eq.(2) at the $t^{\text {th }}$ iteration and $\tau$ is the tolerance parameter. The tolerance is set as $10^{-6}$. All the tests are executed 10 trials and the average measures are used. The initial model parameters of all the three algorithms are given randomly. According to experience of the literature [11], the $\beta_{\max }$ in the DAAEMMM algorithm is set as 1.2. We also test the effect of different $\beta_{\max }=\{1.3,1.4,1.5\}$. At $\beta_{\max }=1.2$, the algorithm always has converged. The 10 runs of the DAAEMMM and DAEMMM algorithms begin with the same $\beta_{0}$, and all the $\beta_{0}$ on different networks are set according to the ones in Table 8. In our DAAEMMM algorithm, we update $\beta$ by $\beta_{\text {new }} \leftarrow \beta_{\text {last }}+0.1$, where $\beta_{\text {new }}$ is $\beta$ in the current iteration and $\beta_{\text {last }}$ is $\beta$ in the last iteration.

\subsection{The validity of the lower bound of $\beta_{0}$}

According to the analysis above, we know the DAAEMMM algorithm should set a proper initial $\beta_{0}$ to avoid meaningless clustering results. According to Eq.(13), the initial $\beta_{0}$ can be determined by the largest eigenvalue (i.e., the convergence rate) at the fixed point

\begin{tabular}{ccccc}
\hline nodes & edges & $\lambda_{\max }$ & $\beta_{\text {theoretical }}$ & $\beta_{0}$ \\
\hline 200 & 3,032 & 3.906 & 0.256 & 0.3 \\
400 & 12,014 & 5.209 & 0.192 & 0.2 \\
800 & 47,832 & 7.743 & 0.129 & 0.2 \\
1600 & 192,203 & 14.282 & 0.070 & 0.1 \\
3200 & 768,240 & 26.722 & 0.037 & 0.1 \\
6400 & $3,073,451$ & 51.041 & 0.019 & 0.1 \\
\hline
\end{tabular}

Table 7: The $\lambda_{\max }$ and $\beta_{\text {theoretical }}$ and $\beta_{0}$ of the DAAEMMM algorithm on synthetic networks with 10 clusters.

$U_{\pi}$ or $U_{1}$. In the following, we simplify the largest eigenvalue $\lambda_{\max }(A)$ of Jacobian matrix by Eq. (13) at $\beta=1$ as $\lambda_{\max }$, and the lower bound $\beta_{\text {theoretical }}$ of $\beta_{0}$ equals to $\frac{1}{\lambda_{\max }}$.

In Table 7 and Table 8 , we show $\lambda_{\max }$, the theoretical lower bound $\beta_{\text {theoretical }}$ of parameter $\beta_{0}$ and real $\beta_{0}$ of the DAAEMMM algorithm on several synthetic and real networks. When $\beta=1$, the convergence rate of the EM algorithm is equal to $\lambda_{\max }$, which should not be less than 1 . As the tables illustrate, all the $\lambda_{\max }$ on all the networks are larger than 1. Results demonstrate that the EM algorithm will not the output meaningless clustering result $U_{\pi}$.

In addition, results in the tables illustrate that different networks have different $\beta_{\text {theoretical }}$. The initial $\beta_{0}$ of the DAAEMMM algorithm should not be less than the corresponding $\beta_{\text {theoretical }}$. The value $\beta_{0}$ of the last columns in Tables 7 and 8 are larger ones than the corresponding $\beta_{\text {theoretical }}$, which are initial $\beta_{0}$ we use in our experiments. If $\beta_{\text {theoretical }}<0.1$, we round up it to nearest tenth and the initial $\beta_{0}=0.1$. This setting not only satisfies $\beta_{0}>\beta_{\text {theoretical }}$, but also makes the algorithm converge faster. If $\beta_{\text {theoretical }}>=0.1$, we also round up it to the nearest tenth and get a larger initial $\beta_{0}$. Several initial $\beta_{0}$ less than the theoretical value have been set for each network, such as 0.1 for the football network and the risk network. They indeed make the DAEMMM algorithm output meaningless partition $[U]_{\frac{1}{c}}$ in the end. Thus, the lower bound $\beta_{\text {theoretical }}$ inferred from our proposed method is valid for the DAAEMMM algorithm.

\subsection{The performance of the DAAEMMM algorithm}

In order to demonstrate the performance of the DAAEMMM algorithm with an appropriate $\beta_{0}$, we execute two kinds of tests. Firstly, we investigate the effec$\mathrm{t}$ of different initial $\beta_{0}$ values above the theoretical value $\beta_{\text {thetoretical }}$ on the dolphin, adjnoun, football networks. 


\begin{tabular}{cccc}
\hline network & $\lambda_{\max }(B)$ & $\beta_{\text {theoretical }}$ & $\beta_{0}$ \\
\hline football & 8.019 & 0.125 & 0.2 \\
lesmis & 10.4341 & 0.096 & 0.1 \\
risk & 3.9961 & 0.25 & 0.3 \\
karate & 6.8265 & 0.1465 & 0.2 \\
dolphin & 6.257 & 0.1598 & 0.2 \\
adjnoun & 6.5188 & 0.1534 & 0.2 \\
polbook & 12.2969 & 0.081 & 0.1 \\
Wiki-Vote & 82.0736 & 0.012 & 0.1 \\
socSlash & 104.774 & 0.01 & 0.1 \\
Web & 175.987 & 0.006 & 0.1 \\
\hline
\end{tabular}

Table 8: The $\lambda_{\max }$ and $\beta_{\text {theoretical }}$ and $\beta_{0}$ of the DAAEMMM algorithm on real networks.

Secondly, we compare the DAAEMMM algorithm with the DAEMMM algorithm and the EMMM algorithm.

Figure 1 illustrates the accuracy of the DAAEMMM algorithm beginning with different $\beta_{0}$ above the theoretical lower bound. It demonstrates that the selected $\beta_{0}$ has the best performance among $\{0.2,0.3,0.4,0.5,0.6,0.7,0.8\}$. According to our parameter selection method, the initial $\beta_{0}$ of the algorithm on the three networks (as Table 8) are all selected as 0.2. For the dolphin network, the DAAEMMM algorithms at the selected $\beta_{0}=$ $\{0.3,0.4,0.5,0.6,0.7,0.8\}$ have the same accuracy as the one at $\beta_{0}=0.2$. That is because the structure of the dolphin network is simple and easy to detect. The adjnoun network is more complex than the dolphin network. The accuracy values at $\beta_{0}=\{0.6,0.7,0.8,0.9\}$ on the adjnoun network are lower than the ones at $\beta_{0}=\{0.2,0.3,0.4,0.5\}$. The football network is the most complex among the three networks. The algorithm at $\beta_{0}=0.2$ has the best performance than other $\beta_{0}$. As the initial $\beta_{0}$ increases, the accuracy value begins to decrease. The reason is that the object function of the complex network has more local maxima, and a larger initial $\beta_{0}$ makes the algorithm easy to converge to local maximum faster. Thus, for the considered initial $\beta_{0}$ values of the complex networks, the performance of the DAAEM algorithm decreases as $\beta_{0}$ increases. In a word, the DAAEM algorithm starting from the selected $\beta_{0}$ according to our parameter selection method has a better performance.

For the second kind of tests, Table 9 shows the result$\mathrm{s}$ of our algorithm and compared algorithms on networks of Table 8. For networks with ground truth, the accuracy

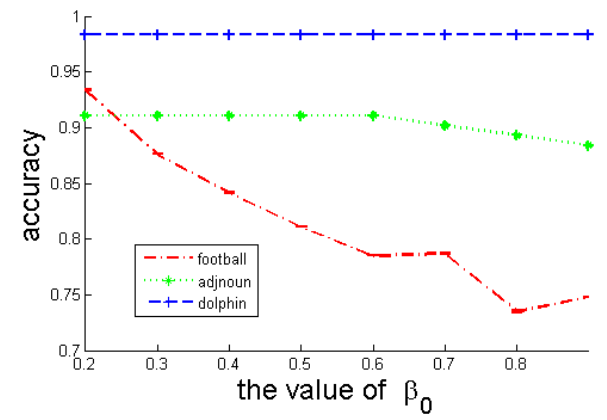

Figure 1: The accuracy of the DAAEMMM algorithm with different $\beta_{0}$ above the $\beta_{\text {thetoretical }}$ value on three networks. Each curve and error bar represent the average accuracy and standard deviation of ten trials, respectively.

\begin{tabular}{cccc}
\hline network & EMMM & DAEMMM & DAAEMMM \\
\hline Wiki-Vote & $-1.108 \times E^{+06}$ & $-1.007 \times E^{+06}$ & $\mathbf{- 1 . 0 0 3} \times E^{+06}$ \\
socSlash & $-2.701 \times E^{+06}$ & $-2.644 \times E^{+06}$ & $\mathbf{- 2 . 5 1 2} \times E^{+06}$ \\
Web & $-2.122 \times E^{+06}$ & $-2.101 \times E^{+06}$ & $\mathbf{- 2 . 0 0 1} \times E^{+06}$ \\
\hline
\end{tabular}

Table 10: Comparisons of the EMMM algorithm and the DAEMMM algorithm and the DAAEMMM algorithm by the average loglikelihood (stand error).

(acc) and Normalized Mutual Information (nmi) are used to measure the algorithms. For networks without ground truth, the log-likelihood is used to measure the algorithms. All the measurements are the average values of results of 10 runs. Results illustrated in the Table 9 and Table 10 show the DAAEMMM algorithm has a better performance. The reason is that the converged log-likelihood of the EMMM algorithm at each run differs largely, and the DAEMMM algorithm also converges to several values in some complex networks, but the log-likelihoods of 10 runs of the DAAEMMM algorithm are approximately equal.

\subsection{Relations between the convergence rate of the DAAEMMM algorithm and the network structure}

In order to judge whether the convergence rate of the DAAEMMM algorithm is affected by the structure of a network, we have tested the algorithm on synthetic networks generated by LFR. In Table 11, all networks have 5000 nodes and assortative mixing are all negative. According to the paper [15], the largest eigenvalue of Jaco- 


\begin{tabular}{ccccccc}
\hline network & EMMM $(\mathrm{acc})$ & DAEMMM $(\mathrm{acc})$ & DAAEMMM $(\mathrm{acc})$ & EMMM $(\mathrm{nmi})$ & DAEMMM $(\mathrm{nmi})$ & DAAEMMM $(\mathrm{nmi})$ \\
\hline football & $0.8087(0.075)$ & $0.9304(0.042)$ & $\mathbf{0 . 9 3 3}(\mathbf{0 . 0 1 2})$ & $0.8546(0.008)$ & $0.9227(0.008)$ & $\mathbf{0 . 9 2 4 9}(\mathbf{0 . 0 1 4})$ \\
lesmis & $0.6234(0.011)$ & $0.7143(0.021)$ & $\mathbf{0 . 7 1 5 5}(\mathbf{0 . 0 0 6})$ & $0.6608(0.009)$ & $0.7208(0.006)$ & $\mathbf{0 . 7 4 5 5}(\mathbf{0 . 0 0 3})$ \\
risk & $0.8055(0.043)$ & $0.8333(0)$ & $\mathbf{0 . 8 6 3 7}(\mathbf{0 . 0 0 3})$ & $0.8571(0.011)$ & $0.9055(0.009)$ & $\mathbf{0 . 9 1 5 2 ( 0 . 0 0 6 )}$ \\
karate & $0.95(0.051)$ & $1(0)$ & $\mathbf{1 ( 0 )}$ & $0.962(0.016)$ & $1(0)$ & $\mathbf{1 ( 0 )}$ \\
dolphin & $0.6652(0.334)$ & $0.9839(0)$ & $\mathbf{0 . 9 8 3 9}(\mathbf{0})$ & $0.753(0.234)$ & $0.9839(0)$ & $\mathbf{0 . 9 8 3 9}(\mathbf{0})$ \\
adjnoun & $0.852(0.023)$ & $0.9018(0.012)$ & $\mathbf{0 . 9 1 1 3 ( 0 . 0 0 7 )}$ & $0.876(0.035)$ & $0.954(0.007)$ & $\mathbf{0 . 9 5 5 ( 0 . 0 0 5 )}$ \\
polbook & $0.8095(0.010)$ & $0.819(0)$ & $\mathbf{0 . 8 2 2}(\mathbf{0 . 0 0 7})$ & $0.5216(0.023)$ & $0.6921(0.015)$ & $\mathbf{0 . 7 0 0 1}(\mathbf{0 . 0 0 5})$ \\
\hline
\end{tabular}

Table 9: Comparisons of the EMMM algorithm and the DAEMMM algorithm and the DAAEMMM algorithm by the average accuracy (stand error) and the average nmi (stand error).

\begin{tabular}{cccccc}
\hline network & $\mathrm{c}$ & edges & assorDegree & conRate & $\beta_{\text {theoretical }}$ \\
\hline net1 & 97 & 99228 & -0.1624 & 26.2899 & 0.038 \\
net2 & 101 & 98186 & -0.162 & 20.2147 & 0.049 \\
net3 & 97 & 97356 & -0.1538 & 16.052 & 0.062 \\
net4 & 95 & 97482 & -0.1343 & 12.1382 & 0.082 \\
net5 & 106 & 98352 & -0.1249 & 9.6437 & 0.103 \\
net6 & 103 & 96930 & -0.1121 & 7.2405 & 0.138 \\
net7 & 94 & 97990 & -0.096 & 5.8761 & 0.170 \\
net8 & 104 & 98096 & -0.084 & 5.0793 & 0.196 \\
net9 & 95 & 97122 & -0.0739 & 5.0624 & 0.197 \\
\hline
\end{tabular}

Table 11: Experiments of relations between assortative mixing by degree and convergence rate on synthetic networks. ' $c$ ' is the number of clusters. 'edges' is the number of edges. 'assorDegree' is assortative mixing by degree. 'conRate' is the convergence rate of the algorithm at $\beta=1$. ' $\beta_{\text {theoretical }}$ ' is the theoretical $\beta_{0}$ by the proposed parameter selection method.

bian matrix $\left.\frac{\partial F^{(\beta)}(p)}{\partial p}\right|_{p^{*}=U_{\pi}}$ can be referred to as the convergence rate at $\beta=1$. Table 11 shows that the larger the assortative mixing by degree, the faster the convergence rate and the larger the initial $\beta_{0}$ of the DAAEMMM algorithm. The results demonstrate that the clearer the structure of a network, the larger the assortative mixing, the faster the convergence rate.

\section{Conclusions}

In order to explore the structure of a network efficiently, the deterministic annealing expectation maximization algorithm is used to estimate parameters of mixture model. A parameter selection method is also provided to select an initial $\beta_{0}$ according to its theoretical lower bound, which is computed by the convergence rate of the algorithm based on Jacobian matrix. As long as $\beta_{0}$ is not less than the lower bound, the DAAEMMM algorithm will not

converge to the defined meaningless results. At the same time, the $\beta_{0}$ we use should not be larger too much than the lower bound. Otherwise the algorithm will converge to local optima more frequently. In our experiments, the $\beta_{0}$ is the value that the lower bound is rounded up to the nearest tenth. Experiments on synthetic networks and real networks demonstrate that the DAAEMMM algorithm and the proposed parameter selection method are valid, and the convergence rate of the DAAEMMM algorithm is affected by the assortative mixing by degree of a network.

So far, we have not seen a similar method that analyzes a DAAEM or DAEM algorithm for network exploring by the convergence rate for parameter selecting tasks. The proposed method also connects the performance of the algorithm in a network and the structure of a network with the convergence rate of the algorithm. It is easy to be extended to other algorithms for community detection. In the future, we will further study other algorithms for community detection based on our proposed method.

\section{Acknowledgments}

This work is supported by the National Science Foundation (Grant No. 61503260), the open project of Beijing Key Lab of Traffic Data Analysis and Mining, the Hebei Province Science and Technology Planed Projects (Grant No.14214504D), the Natural Science Foundation of Hebei Province (Grant No. F2013205192) . The authors thank the anonymous reviewers for their constructive comments. 


\section{References}

[1] Santo Fortunato. Community detection in graphs. Physics Reports, 486(3):75-174, 2010.

[2] Paul W Holland, Kathryn Blackmond Laskey, and Samuel Leinhardt. Stochastic blockmodels: First steps. Social Networks, 5(2):109-137, 1983.

[3] T.A.B. Snijders and K. Nowicki. Estimation and prediction for stochastic blockmodels for graphs with latent block structure. Journal of Classification, 14(1):75-100, 1997.

[4] J.J. Daudin, F. Picard, and S. Robin. A mixture model for random graphs. Statistics Computing, 18(2):173-183, 2008.

[5] Hua Wei Shen, Xue Qi. Cheng, and Jia Feng Guo. Exploring the structural regularities in networks. Physical Review E, 84(5):056111, 2011.

[6] Bian fang Chai, Jian Yu, Cai yan Jia, Tian bao Yang, and Ya wen Jiang. Combining a popularity-productivity stochastic block model with a discriminative-content model for general structure detection. Physical Review E, 88(1):012807, 2013.

[7] Jake M Hofman and Chris H Wiggins. Bayesian approach to network modularity. Physical Review Letters, 100(25):258701, 2008.

[8] Aurelien Decelle, Florent Krzakala, Cristopher Moore, and Lenka Zdeborová. Inference and phase transitions in the detection of modules in sparse networks. Physical Review Letters, 107(6):065701, 2011.

[9] MEJ Newman and EA Leicht. Mixture models and exploratory analysis in networks. Proceedings of the National Academy of Sciences, 104(23):9564, 2007.

[10] Naonori Ueda and Ryohei Nakano. Deterministic annealing em algorithm. Neural Networks, 11(2):271-282, 1998.

[11] Iftekhar Naim and Daniel Gildea. Convergence of the em algorithm for gaussian mixtures with unbalanced mixing coefficients. In Proceedings of the 29th International Conference on Machine Learning, 2012.
[12] Ulrike Von Luxburg and Shai Ben David. Towards a statistical theory of clustering. In Pascal workshop on statistics and optimization of clustering, 2005.

[13] Lei Xu and Michael I Jordan. On convergence properties of the em algorithm for gaussian mixtures. Neural computation, 8(1):129-151, 1996.

[14] Jian Yu, Qiansheng Cheng, and Houkuan Huang. Analysis of the weighting exponent in the fcm. IEEE Transactions on Systems, Man and Cybernetics-part B: Cybernetics, 34:634-639, 2004.

[15] Chaomurilige, Jian Yu, and Miin Shen Yang. Analysis of parameter selection for gustafson-kessel fuzzy clustering using jacobian matrix. IEEE Transactions on Fuzzy Systems, 23(6):2329-2342, 2015.

[16] CF Jeff Wu. On the convergence properties of the em algorithm. The Annals of statistics, pages 95103, 1983.

[17] Jinwen Ma, Lei Xu, and Michael I Jordan. Asymptotic convergence rate of the em algorithm for gaussian mixtures. Neural Computation, 12(12):28812907, 2000.

[18] Jinwen Ma and Shuqun Fu. On the correct convergence of the em algorithm for gaussian mixtures. Pattern Recognition, 38(12):2602-2611, 2005.

[19] Peter.J Olver. Lecture notes on numerical analysis. http://www.math.umn.edu/ olver/num.html, 2008.

[20] Andrea Lancichinetti, Santo Fortunato, and Filippo Radicchi. Benchmark graphs for testing community detection algorithms. Physical Review E, 78(4):046110, 2008. 